\title{
Étude préliminaire des effets à court et long terme d'un dispositif de formation à la communication d'une mauvaise nouvelle
}

\author{
Short and long terms effects of a training workshop on breaking bad news
}

Tony IBRAHIM ${ }^{1}$, Georges MAALOULY ${ }^{1}$, Elie NEMR ${ }^{1}$, Elie HADDAD ${ }^{1}$, Charbel YAZBECK ${ }^{2}$, Fady HADDAD ${ }^{1}$

1 CHU Hôtel Dieu de France, Université Saint-Joseph, Beyrouth, Liban

2 CHU Notre Dame des Secours, Université Saint-Esprit de Kaslik, Liban

Manuscrit reçu le 11 octobre 2012 ; commentaires éditoriaux formulés aux auteurs le 9 mars, le 11 août et le 14 août 2013 ; accepté pour publication le 14 août 2013.

\begin{abstract}
Mots-clés communication médicale ; annonce d'une mauvaise nouvelle ; formation ; ECOS ; évaluation

Résumé-Introduction : L'expérience de son problème de santé par un malade est affectée aussi bien par les actes du médecin que par son attitude. L'annonce d'une mauvaise nouvelle est un défi pour les médecins. Objectif : La présente étude a pour objectif de mesurer les effets d'un atelier de formation aux techniques de communication dans le cadre de l'annonce d'une mauvaise nouvelle, en recourant à une évaluation écrite à court et à long terme et à un dispositif de simulation répondant au format de deux stations d'examen clinique objectif standardisé(ECOS), chez des résidents du tronc commun de médecine interne au Liban. Matériels et méthodes : L'étude rapportée est une étude interventionnelle, comparative et contrôlée, évaluant à l'aide de mesures à court et à long termes les effets du dispositif sur les apprentissages de 21 résidents comparativement à celui de 27 résidents d'un groupe contrôle. L'atelier comporte trois étapes réparties sur une durée totale de quatre heures. La méthode d'évaluation comportait des mesures effectuées lors de trois étapes théoriques/écrites (test pré-intervention avant l'atelier et tests postintervention à trois mois et à deux ans) et d'une étape pratique grâce à un examen clinique objectif et structuré (ECOS), effectué trois mois après l'atelier par un évaluateur aveugle écoutant l'enregistrement sonore des entretiens. Résultats : Une amélioration théorique a été constatée chez les membres du groupe atelier (scores de 11,2, 13 et 14,1 pour les moyennes respectives du test pré-intervention, du test post-intervention à trois mois et du test post-intervention à deux ans ; $p<0,05)$ mais pas chez le groupe contrôle. Il n'y a pas de différence concernant l'évaluation pratique par deux stations de type ECOS entre les deux groupes. Discussion : Selon notre étude, l'atelier de formation a pu améliorer l'apprentissage théorique mais pas la pratique de l'annonce d'une mauvaise nouvelle. Conclusion : Un atelier de formation à la communication a un impact positif sur les connaissances en matière d'annonce d'une mauvaise nouvelle mais ses conséquences sur l'acquisition d'authentiques compétences restent à documenter.
\end{abstract}




\section{Keywords}

Breaking bad new; learning; OSCE; evaluation; medical communication
Abstract - Introduction: What physicians do, what they say and how they behave affect how patients experience their health issues. Passing on bad news is a challenge for medical staff. Objective: The aim of the study is to establish the usefulness of a workshop designed to provide the skills in passing on bad news. Materials and methods: This interventional study compared a workshop group of 21 residents to a control group of 27 residents. The workshop was comprised of three stages spread over four hours. The evaluation method involved a theoretical evaluation made up of 3 questionnaires (a pre-intervention test before the workshop, and two post-intervention tests, at 3 months and 2 years after the workshop) and an Objective Structured Clinical Exam (OSCE) 3 months after workshop through a blind assessment of the audio recording. Results: Significant improvement was noted in the theoretical evaluation of the workshop group $(11.2,13$ and 14.1 respectively for the pre-intervention test, post-intervention test at 3 months and post-intervention test at 2 years, with a $p$ value $<0.05$ ) but not of the control group. No variation was noted in the OSCE evaluation between the two groups. Discussion: Based on this study, the communication skills workshop had improved the theoretical side of learning in passing on bad news but not the practical side. The limited length of the single workshop and the cultural differences of participants may provide an explanation for these results. Conclusion: A communication skills workshop has a positive impact on knowledge about strategies on how to pass on bad news.

\section{Introduction}

Dans le contexte d'une médecine de plus en plus technique et performante, la relation médecin-malade a connu de profondes modifications au cours des dernières décennies. Cependant, au-delà des aspects bio-techniques, la communication reste un déterminant important de la satisfaction du malade et de sa compliance au traitement ${ }^{[1]}$. Ainsi, dès 1847 , la première version du code d'éthique de l'Association médicale américaine (citée par Vandekieft ${ }^{[2]}$ ), stipulait déjà que « la vie d'une personne malade peut être abrégée autant par la parole que par actes et les comportements du médecin ». Lors de l'annonce d'une mauvaise nouvelle, sont notamment sollicitées les compétences de communication ; une telle situation est un défi pour les médecins qui doivent faire face aux réactions émotionnelles du patient, au stress créé par ses attentes et aux difficultés de l'alliance thérapeutique dans ces conditions ${ }^{[3,4]}$. Ainsi en oncologie clinique, la capacité de communiquer efficacement avec le patient et sa famille n'est plus considérée comme facultative mais comme une nécessité ${ }^{[5]}$. Les recommandations actuelles de l'Association américaine des facultés de médecine (Association of American Medical Colleges - AAMC), font désormais de l'entrainement aux techniques de communication un élément essentiel de la formation ${ }^{[3]}$. Dans le sillage du processus de Bologne, le projet de l'European Core Curriculum ${ }^{[6]}$ a par ailleurs défini de manière similaire les neuf champs de compétences suivants : les compétences cliniques, les capacités de communication, la pensée critique, la prise en compte des objectifs de santé publique, la formation continue, le professionnalisme, l'enseignement, le travail en équipe et les connaissances théoriques. De fait, l'apprentissage de la capacité à annoncer de façon efficiente une mauvaise nouvelle est essentiel pour être en mesure de fournir de bons soins médicaux, maintenir une relation productive avec le malade, et satisfaire aussi bien le médecin que le malade ${ }^{[7]}$.

Plusieurs cadres opérationnels de dispositifs pédagogiques dédiés à la formation à l'annonce d'une mauvaise nouvelle ont été développés, souvent désignés par des acronymes à visée mnémonique, tels que le guide $\mathrm{ABCDE}^{[8]}$ (respectivement pour : Advance preparation ; $\underline{B}$ uild a therapeutic environment/relationship ; Communicate well; Deal with patient and family reactions; Encourage and validate emotions) ou encore le modèle SPIKES ${ }^{[3]}$ (respectivement pour: $\underline{\text { Setting }}$ up the interview -aménager l'entretien-, assessing the patient's Perception -évaluer la perception du malade-, obtaining the patient's Invitation -solliciter le consentement du 
patient-, giving Knowledge and information to the patient -informer le patient-, adressing the patient's Emotion with Empathic responses - prendre en compte les émotions du patient grâce à des réponses empathiques- et $\underline{\text { Strategy and } \underline{S} u m m a r y ~-p r e ́ s e n t e r ~ l a ~}$ stratégie de traitement et la résumer. Ce denier est un guide opératoire en six étapes, développé en contexte nord-américain et exploité par plusieurs facultés de médecine de par le monde ${ }^{[9,10,11,12]}$.

Une revue de littérature réalisée par Rosenbaum et al. ${ }^{[9]}$ montre que les dispositifs de formation à l'annonce d'une mauvaise nouvelle qui exploitent ce modèle améliorent la satisfaction et la confiance des participants indépendamment de la méthode d'enseignement. Les effets et l'impact de tels dispositifs restent cependant encore peu documentés; ainsi, le nombre d'études ayant cherché à évaluer l'impact d'un atelier d'enseignement grâce à des mesures objectives, par exemple en recourant à un évaluateur aveugle grâce à des enregistrements sonores de rencontres médecin-patient ou encore à des patients standardisés, est limité ${ }^{[13]}$. Celles qui sont disponibles ont montré une amélioration significative des modalités d'annonce de la mauvaise nouvelle ${ }^{[13,14,15,16]}$. La plupart ont en outre été réalisée en milieu anglosaxon ; à notre connaissance aucune étude similaire mesurant l'impact à long terme de tels ateliers n'a été réalisée dans le cadre de curriculums de formation médicale développés en contexte arabe.

Notre étude a pour objectif de mesurer les effets d'un atelier de formation aux techniques de communication dans le cadre de l'annonce de la mauvaise nouvelle, en recourant à une évaluation écrite à court et à long terme et à un dispositif de simulation répondant au format de deux stations d'examen clinique objectif standardisé (ECOS), chez des résidents du tronc commun de médecine interne au Liban.

\section{Matériels et méthodes}

\section{Population de l'étude et échantillon}

Nous rapportons une étude interventionnelle, comparative, contrôlée, menée à l'université Saint-Joseph à Beyrouth (Liban) entre les mois de juillet 2008 et décembre 2011. La population potentiellement concernée était constituée de l'ensemble des résidents inscrits dans le tronc commun de médecine interne de l'université Saint-Joseph (première, deuxième et troisième année de spécialisation postgraduée). Cependant, par convenance, seuls les résidents en stage hospitalier au centre hospitalier universitaire de l'Hôtel Dieu de France (CHU-HDF) de Beyrouth ont été étudiés. Les sujets ont été invités à participer par message électronique expliquant en détails l'atelier. Parmi 50 résidents répondant aux critères d'inclusion, 48 ont participé et ont signé un consentement écrit, deux n'ayant pu participer pour des raisons de déplacement.

Les participants ont été divisés en deux groupes d'une façon aléatoire. Le premier groupe (groupe atelier) comprenait les 21 sujets participant à la formation. Le second groupe (groupe contrôle) comprenait les 27 sujets qui n'ont pas bénéficié de la formation.

\section{Intervention pédagogique}

L'atelier de formation est composé de trois étapes : la première est un cours magistral de 60 minutes avec projection d'un support écrit sous forme de diaporama, élaboré par l'un des auteurs, expliquant le modèle SPIKES. La deuxième étape, d'une durée de 120 minutes, est faite de jeux de rôle réalisés par les résidents eux-mêmes, proposant l'exploration expérientielle de situations auxquelles ils se sont confrontés lors de leurs stages, avec discussion de chaque cas. La dernière étape est constituée par la présentation d'une vidéo de 60 minutes, mettant en scène plusieurs scénarios d'annonce de mauvaises nouvelles dans un cadre clinique.

Données recueillies et mesures effectuées

\section{Données descriptives}

L'âge, le sexe et l'année de résidanat de chaque étudiant ont été colligées. Par ailleurs, pour chacun d'entre eux, a été déterminée une variable 
psychologique de personnalité qui permet de préciser la manière selon laquelle ils appréhendent le contrôle des situations auxquelles ils sont exposés : l'échelle de Rotter concerne plus particulièrement la dimension cognitive du contrôle et permet de mesurer la croyance des individus à l'égard de ce qui détermine leur réussite dans une activité particulière. Les personnes croyant que leur performance dépend surtout d'eux-mêmes ont un locus de contrôle dit « interne »; celles persuadées que l'issue est avant tout déterminée par des facteurs extérieurs, hors de leur influence, ont un locus de contrôle dit «externe ${ }^{[17]}$. Par cette mesure, il s'agissait de vérifier si les deux groupes étaient comparables au regard d'une disposition psychologique importante en termes de profil cognitivo-comportemental et de dynamique d'apprentissage.

\section{Pré-test et post-tests administrés par écrit}

Avant l'atelier, un test pré-intervention, composé de questions explorant respectivement différentes situations cliniques présentées sous la forme d'un court énoncé, a été administré par écrit. Il a été élaboré en langue française par les auteurs de l'étude à partir du questionnaire de l'étude de Farber et al. ${ }^{18]}$ (Cf. Annexe). Chacune des 21 situations fait l'objet d'une question à choix de réponse parmi trois à quatre possibles, le participant étant invité à sélectionner celle qu'il considérait comme étant la meilleure. Ce questionnaire a été préalablement testé par une étude exploratoire ayant inclus 10 médecins, de manière à vérifier sa compréhensibilité puis il a été administré pour la première fois aux deux groupes, simultanément, avant l'atelier pour être rempli en 126 minutes (soit 6 minutes par cas).

Respectivement trois mois (test postintervention 1) et deux ans (test post-intervention 2) après l'atelier, le même questionnaire a été ré-administré aux deux groupes.

\section{Post-test de type ECOS}

Après le test écrit post-intervention 1, d'autre part, les membres des deux groupes ont été exposés à un exercice de simulation constitué de deux stations
d'ECOS. Chacune, d'une durée de 15 minutes, consistait en un scénario, inspiré de la réalité, durant lequel le participant devait annoncer une mauvaise nouvelle à un patient simulé standardisé, dont le rôle était joué par des personnes entrainées à l'ECOS et ne faisant pas partie du corps médical. Ces acteurs avaient également pour fonction d'évaluer les performances de communication non verbale des participants, en fournissant un score sur une échelle de 10 points, non assortie de descripteur qualitatif. Un enregistrement sonore de l'entretien était réalisé. Un évaluateur « aveugle » unique (ne connaissant pas les participants), médecin formé à la pédagogie médicale dans le cadre d'un diplôme universitaire, évaluait les entretiens enregistrés de l'ensemble des participants à l'aide de la grille d'évaluation utilisée dans l'étude d'Alexander et al. ${ }^{[13]}$. Cette grille évalue les paramètres suivants: A) Communication de la mauvaise nouvelle : 1) introduire l'entretien par une phrase préparatoire ; 2) annoncer la mauvaise nouvelle tôt dans la conversation ; 3) utiliser un langage clair et explicite ; 4) rester silencieux après l'annonce de la mauvaise nouvelle ; 5) chercher à évaluer la réaction du patient envers la mauvaise nouvelle. B) Réponse aux émotions du patients : 1) définir l'émotion du patient ; 2) chercher les émotions non exprimées explicitement par le patient ; 3) exprimer la compréhension envers l'émotion du patient ; 4) chercher à explorer les facteurs qui influencent l'émotion du patient. C) Compétence générale de communication : 1) établir le rapport initial avec le patient ;2) chercher à évaluer le soutien social du patient ; 3) informer le patient du soutien médical continu de la part du médecin; 4) vérifier si le patient a des questions à formuler; 5) vérifier que le patient est dans de bonnes conditions pour prendre des décisions ; 6) faire un résumé de tout ce qui a été dit et du plan d'action médicale future.

L'ensemble des interventions et mesures effectuées dans le cadre du protocole d'étude est récapitulé dans le tableau I.

\section{Analyse statistique}

L'analyse statistique a été réalisée avec le logiciel PASW version 18 (Predictive Analytics SoftWare). Les données descriptives sont présentées sous forme 
Tableau I. Récapitulatif du protocole méthodologique de l'étude.

\begin{tabular}{|l|l|l|}
\hline \multicolumn{2}{|l|}{ Nature de l'intervention ou de la mesure } & Durée \\
\hline \multicolumn{2}{|l|}{ Test pré-intervention } & 126 minutes \\
\hline Atelier de formation & Conférence orale non interactive & 60 minutes \\
\cline { 2 - 3 } & Jeu de rôle interactif & 120 minutes \\
\cline { 2 - 3 } & Vidéo & 60 minutes \\
\hline Test post-intervention 1 (+ 3 mois) & 126 minutes \\
\hline Deux stations ECOS (+ 3 mois) & $\begin{array}{l}\text { Deux situations simulées } \\
\text { standardisées de 30 minutes chacune }\end{array}$ \\
\hline Test post-intervention 2 (+ 2 ans) & 126 minutes \\
\hline
\end{tabular}

de moyennes et de fréquences. La comparaison entre les deux groupes (groupe atelier vs. groupe contrôle) a été réalisée grâce à un test de chi-deux $(\chi 2)$ avec le risque d'erreur de premier degré alpha bilatéral fixé à $5 \%$. pour les variables suivantes : sexe, année de résidanat, locus de contrôle mesuré par l'échelle de Rotter.

La comparaison des moyennes (âge, scores aux tests pré- et post-intervention à trois mois et deux ans, score à l'ECOS à trois mois) a été réalisée grâce aux test de Student (pour les échantillons indépendants ou pour les séries appariées, selon le cas) en spécifiant le risque d'erreur de premier degré alpha bilatéral fixé à $5 \%$ et cela après avoir tester l'homogénéité des variances par un test ANOVA et la normalité de distribution des notes par un test de Shapiro-Wilk.

L'analyse de corrélation partielle entre l'appartenance à un groupe (atelier ou contrôle) et les scores de l'ECOS scenario 1 et ECOS scenario 2, en contrôlant les variables âge, sexe, année de résidanat et score de Rotter a été calculée avec le risque d'erreur de premier degré alpha bilatéral fixé à $5 \%$.

\section{Résultats}

Les caractéristiques démographiques, éducatives et psychologiques des participants à l'étude ainsi que les résultats du test pré-intervention sont résumés dans le tableau II. Aucune différence statistiquement significative n'a été trouvée entre le groupe atelier et le groupe contrôle concernant les variables âge, sexe, année de résidanat, score de Rotter et les moyennes du test pré-intervention. En analysant la suite des résultats (tableau III), une différence statistiquement significative a été trouvée dans le groupe atelier entre le test pré-intervention et le test post-intervention à trois mois (amélioration relative de $14 \%: 11,2$ contre 13 respectivement) et à deux ans (amélioration relative du score de près de $26 \%: 11,2$ contre 14,1 respectivement) mais pas entre le test postintervention à trois mois et le test post-intervention à deux ans. Il n'a pas été trouvé de différence significative entre le test pré-intervention et le test postintervention dans le groupe contrôle. La moyenne du test post-intervention du groupe atelier a été trouvée significativement supérieure à celle du groupe contrôle (13 contre 11 respectivement, avec une valeur $p<0,05)$. D'autre part aucune différence n'a été trouvée entre les deux groupes pour les scores des deux stations d'ECOS, aussi bien pour le score total que les sous-scores (tableau IV). L'étude de l'influence du sexe sur le résultat du test écrit et des scores aux deux stations ECOS n'a pas montré de différence entre les hommes et les femmes au sein des deux groupes (résultat non fourni). De plus, une corrélation partielle a été établie entre les scores aux stations ECOS et l'appartenance à un des deux groupes, en contrôlant les autres variables démographiques (âge, sexe), éducatives (années résidanat) et psychologiques (échelle de Rotter). Le résultat résumé dans le tableau $\mathrm{V}$ ne montre une relation significative qu'avec le score total obtenu au deuxième scenario d'ECOS (coefficient de corrélation : $-0,38 ; p=0,048)$. 
Tableau II. Comparaison des variables âge, sexe, année de résidanat, score à l'échelle de Rotter et score au test préintervention entre le groupe atelier et le groupe contrôle.

\begin{tabular}{|c|c|c|c|c|}
\hline & & $\begin{array}{c}\text { Groupe atelier } \\
\qquad N=21\end{array}$ & $\begin{array}{c}\text { Groupe contrôle } \\
\qquad N=27\end{array}$ & Valeur $p$ \\
\hline \multicolumn{2}{|c|}{ Moyenne (Déviation Std.) } & $26(2)$ & $25(1,3)$ & $>0,05^{\mathbf{a}}$ \\
\hline \multirow{2}{*}{$\begin{array}{l}\text { Sexe } \\
\text { Effectifs }(\%)\end{array}$} & Femmes & $13(61,9 \%)$ & $15(55,5 \%)$ & \multirow[t]{2}{*}{$>0,05^{\mathbf{b}}$} \\
\hline & Hommes & $8(38,1 \%)$ & $12(44,5 \%)$ & \\
\hline Années résidanat & R1 & $13(61,9 \%)$ & $18(66,7 \%)$ & \multirow{3}{*}{$>0,05^{\mathbf{b}}$} \\
\hline \multirow{2}{*}{ Effectifs $(\%)$} & $\mathbf{R 2}$ & $5(23,8 \%)$ & $6(22,2 \%)$ & \\
\hline & $\mathbf{R 3}$ & $3(14,3 \%)$ & $3(11,1 \%)$ & \\
\hline \multirow[t]{3}{*}{$\begin{array}{l}\text { Echelle de Rotter } \\
\text { Effectifs }(\%)\end{array}$} & $\begin{array}{l}\text { Locus de contrôle interne } \\
\text { extrême. } \\
\text { Echelle de Rotter : } 0-3\end{array}$ & $3(14,2 \%)$ & $3(11,2 \%)$ & \multirow{3}{*}{$>0,05^{\mathbf{b}}$} \\
\hline & $\begin{array}{l}\text { Locus de contrôle interne } \\
\text { modéré. } \\
\text { Echelle de Rotter : 4-11 }\end{array}$ & $11(52,4 \%)$ & $14(51,8 \%)$ & \\
\hline & $\begin{array}{l}\text { Locus de contrôle externe. } \\
\text { Echelle de Rotter : } 12-23\end{array}$ & $7(33,4 \%)$ & $10(37,0 \%)$ & \\
\hline \multicolumn{2}{|l|}{ Pré-test } & $11,14(1,982)$ & $10,35(2,432)$ & $>0,05^{\mathbf{a}}$ \\
\hline
\end{tabular}

$\mathbf{a}=$ analyse statistique utilisant le test $\mathrm{t}$ de Student pour la comparaison des moyennes entre des échantillons indépendants.

$\mathbf{b}=$ analyse statistique utilisant le test de $\chi^{2}$ (chi-2) pour tester l'hétérogénéité de la distribution des effectifs entre les groupes.

Le test de normalité de distribution (test de Shapiro-Wilk) ainsi qu'un test d'homogénéité des variances (Levene's test) ont été réalisés avant la comparaison des moyennes avec $p>0,05$, conditions nécessaires pour l'application du test de Student.

\section{Discussion}

L'annonce d'une mauvaise nouvelle est un élément important affectant la relation médecin-patient, surtout en contexte de soins palliatifs ou même de certaines maladies à pronostic réservé ${ }^{[20-23]}$. Un médecin non formé éprouve des difficultés à annoncer une mauvaise nouvelle, ce qui a des conséquences en termes d'émotion du patient et de stress créé par ses attentes ${ }^{[3]}$. Le modèle SPIKES est un modèle opératoire simple, qui propose que la communication d'une mauvaise nouvelle puisse répondre à un processus en plusieurs étapes essentielles ${ }^{[3,12]}$. Divers dispositifs pédagogiques exploitant ce modèle ont été utilisées pour enseigner l'annonce d'une mauvaise nouvelle, tels que des conférences magistrales, des scénarios cliniques, ainsi que des discussions en groupe ${ }^{[9,10,13,24,25,26]}$. Notre étude concerne un dispositif pédagogique réalisé sous la forme d'un atelier qui associe trois types d'intervention. Le cours magistral permet de présenter de manière théorique une méthode recommandée pour l'annonce d'une 
Tableau III. Comparaison entres les scores obtenus respectivement aux test pré-intervention, test post-intervention à trois mois et à deux ans au sein des groupes « contrôle » et « atelier ».

\begin{tabular}{|c|c|c|c|c|}
\hline Groupe atelier & $\mathrm{N}$ & Moyenne & Déviation Standard. & Valeur $p$ \\
\hline Test pré-intervention & 21 & 11,2 & 2,2 & $<0,05^{* a}$ \\
\hline Test post-intervention & 21 & 13 & 1,9 & $<0,05^{* \mathbf{b}}$ \\
\hline $\begin{array}{l}\text { Test post-intervention } \\
\text { à deux ans }\end{array}$ & 21 & 14,1 & 1,6 & $>0,05^{\mathbf{c}}$ \\
\hline \multicolumn{5}{|l|}{ Groupe contrôle } \\
\hline Test pré-intervention & 27 & 10,3 & 2,5 & $>0,05^{\mathbf{a}}$ \\
\hline Test post-intervention & 27 & 10,4 & 2,8 & $>0,05^{\mathbf{b}}$ \\
\hline $\begin{array}{l}\text { Test post-intervention } \\
\text { à deux ans }\end{array}$ & 27 & 10,2 & 2,1 & $>0,05^{\mathrm{c}}$ \\
\hline
\end{tabular}

$\mathbf{a}=$ test $\mathrm{t}$ de Student des séries appariées pour comparer le pré-test $v s$. post-test

$\mathbf{b}=$ test $t$ de Student des séries appariées pour comparer le pré-test $v s$. post-test après deux ans.

$\mathbf{c}=$ test $\mathrm{t}$ de Student des séries appariées pour comparer le post-test $1 \mathrm{vs}$. post-test 2 après deux ans

* Différence entre les moyennes est statistiquement significative.

Le test de normalité de distribution (test de Shapiro-Wilk) ainsi qu'un test d'homogénéité des variances (Levene's test) ont été réalisés avant la comparaison de chaque paire de groupe montrant des valeurs $p>0,05$, conditionsnécessaire pour l'utilisation du test de Student.

mauvaise nouvelle selon le modèle SPIKES. La séance de discussion en petits groupes permet d'identifier les difficultés qu'ont les participants à annoncer une mauvaise nouvelle et de rendre explicites les erreurs les plus fréquentes. Enfin, la présentation d'une vidéo permet de mieux illustrer ces erreurs.

Les résultats montrent que cet atelier a été efficace pour ce qui concerne les apprentissages théoriques, évalués par un questionnaire écrit, en montrant une amélioration statistiquement significative du score trois mois après l'atelier, entre le groupe atelier et le groupe contrôle (tableau II). Cette amélioration s'est maintenue au terme de deux ans, suggérant que les connaissances déclaratives induites dans le cadre de cet atelier sont pérennes. En revanche, il n'a pas été trouvé d'amélioration significative du score lors de l'évaluation pratique dans le cadre de deux stations de type ECOS (tableau III). Bien qu'une corrélation significative ait été trouvée entre le score total aux deux stations ECOS et l'appartenance au groupe contrôle, après contrôle des autres variables (tableau IV), ceci ne suffit pas pour conclure que le dispositif pédagogique mis en œuvre dans le cadre de l'étude induise des apprentissages responsables d'une amélioration de la pratique de l'annonce d'une mauvaise nouvelle. Pourtant, l'étude d'Alexander et al. ${ }^{[13]}$ avait mis en évidence une amélioration significative des scores obtenus lors de tests de type ECOS. Cette différence pourrait être expliquée par le fait que l'atelier mis en place dans le cadre de cette étude (16 heures) avait une durée largement supérieure au nôtre. Ceci pourrait souligner la nécessité que les dispositifs de formation à la communication d'une mauvaise nouvelle doivent, indépendamment de leurs modalités pédagogiques, être développés sur une durée minimale. Certains auteurs considèrent d'ailleurs que des séances de consolidation de l'apprentissage sont nécessaires pour optimiser la pratique de l'annonce d'une mauvaise nouvelle ${ }^{[27,28]}$. Par ailleurs, ces discordances de résultats pourraient 
Tableau IV. Comparaison des scores obtenus respectivement au test post-intervention, à l'ECOS1 et à l'ECOS 2 entre les deux groupes « contrôle » et « atelier ».

\begin{tabular}{|c|c|c|c|c|}
\hline & & $\begin{array}{c}\text { Groupe atelier } \\
\text { Moyenne } \\
\text { (Déviation } \\
\text { Standard) }\end{array}$ & $\begin{array}{c}\text { Groupe contrôle } \\
\text { Moyenne } \\
\text { (Déviation } \\
\text { Standard) }\end{array}$ & $\begin{array}{l}\text { Test t entre } \\
\text { les échantillons } \\
\text { indépendants- } \\
\text { valeur } p\end{array}$ \\
\hline \multicolumn{2}{|c|}{ Test post-intervention } & $13(1,9)$ & $11(2,8)$ & $<0,05^{*}$ \\
\hline \multirow[t]{4}{*}{ ECOS scenario 1} & $\begin{array}{l}\text { † Compétence de } \\
\text { communication Générale } \\
\text { (note sur } 12 \text { ) }\end{array}$ & $5(1,0)$ & $5(1,8)$ & $>0,05$ \\
\hline & $\begin{array}{l}\$ \text { Annonce } \\
\text { de la mauvaise nouvelle } \\
\text { et réponse aux émotions } \\
\text { du patient (note sur 9) }\end{array}$ & $5(1,1)$ & $5(1,9)$ & $>0,05$ \\
\hline & $\begin{array}{l}\text { Communication non } \\
\text { verbale (note sur } 10 \text { ) }\end{array}$ & $7,4(1,1)$ & $6,7(1,7)$ & $>0,05$ \\
\hline & $\begin{array}{l}\text { Score total } \\
\text { de communication } \\
\text { verbale (note sur } 21)\end{array}$ & $10(1,9)$ & $10(3,1)$ & $>0,05$ \\
\hline \multirow[t]{4}{*}{ ECOS scenario 2} & $\begin{array}{l}\text { Compétence de } \\
\text { communication } \\
\text { générale (note sur 12) }\end{array}$ & $6,5(1,2)$ & $5,9(1,3)$ & $>0,05$ \\
\hline & $\begin{array}{l}\text { Annonce de la mauvaise } \\
\text { nouvelle et réponse } \\
\text { aux émotions du patients } \\
\text { (note sur 9) }\end{array}$ & $5(1,3)$ & $5(1,4)$ & $>0,05$ \\
\hline & $\begin{array}{l}\text { Communication non } \\
\text { verbale (note sur } 10 \text { ) }\end{array}$ & $7.0(1,0)$ & $7.1(1,5)$ & $>0,05$ \\
\hline & $\begin{array}{l}\text { Score total de } \\
\text { communication verbale }\end{array}$ & $12(2,0)$ & $11(2,1)$ & $>0,05$ \\
\hline
\end{tabular}

* Différence entre les moyennes est statistiquement significative.

$\dagger$ Score de 0 (aucune technique n'a été utilisée) à 12 (toutes les six techniques ont été utilisées) soit 2 points par techniques.

† Score de 0 (aucune technique n'a été utilisée) à 9 (toutes les neuf techniques ont été utilisées.

Le test de normalité de distribution (test de Shapiro-Wilk) ainsi qu'un test d'homogénéité des variances (Levene's test) ont été réalisés avant la comparaison des moyennes montrant des valeurs $p>0,05$, conditions nécessaire pour l'utilisation du test de Student.

ECOS = examen clinique objectif structuré.

être dues à la différence culturelle entre les participants des différentes études, d'autant que le modèle SPIKES a été construit par des équipes américaines sur la base de leur expérience avec des patients issus d'un milieu culturel différent de celui des participants de notre étude.
Les caractéristiques du dispositif pédagogique mis en place dans le cadre de notre étude méritent aussi d'être questionnées au regard de leur capacité à susciter des apprentissages réellement transférables. D'une certaine manière, il est en effet surtout centré sur l'apprentissage d'habiletés et de techniques 
Tableau V. Analyse de corrélation partielle entre l'appartenance à un groupe (atelier ou contrôle) et les scores de l'ECOS1 et ECOS 2, en contrôlant les variables âge, sexe, années résidanat et échelle de Rotter.

\begin{tabular}{|c|c|c|c|c|c|c|c|c|}
\hline \multirow{2}{*}{$\begin{array}{l}\text { Corrélations } \\
\text { partielle avec la } \\
\text { variable suivante } \\
\text { «Groupe » } \dagger\end{array}$} & \multicolumn{4}{|c|}{ ECOS groupe atelier } & \multicolumn{4}{|c|}{ ECOS groupe contrôle } \\
\hline & CG & AMN & $\mathrm{CNV}$ & $\begin{array}{l}\text { Score } \\
\text { total }\end{array}$ & CG & AMN & $\mathrm{CNV}$ & $\begin{array}{l}\text { Score } \\
\text { total }\end{array}$ \\
\hline $\begin{array}{l}\text { Coefficient de } \\
\text { corrélation }\end{array}$ & $-0,214$ & $-0,027$ & $-0,222$ & $-0,154$ & $-0,275$ & $-0,225$ & 0,081 & $-0,380$ \\
\hline Valeur $p$ & 0,284 & 0,892 & 0,267 & 0,443 & 0,164 & 0,259 & 0,688 & $0,049 *$ \\
\hline
\end{tabular}

plutôt que sur celui d'authentiques compétences, réellement mobilisables à l'intérieur d'une famille de situations. En outre, bien qu'il recoure à des jeux de rôle, on peut considérer que le dispositif est assez conventionnel puisqu'il débute par une séquence de cours magistral et que la troisième et dernière étape, bien que recourant à la vidéo, est assez transmissive et démonstrative, impliquant peu les étudiants. D'une façon générale, on peut considérer que les conditions pédagogiques mises en œuvre visant à favoriser le transfert pourraient être optimisées.

Il convient par ailleurs de s'interroger sur les limites des dispositifs de mesure utilisés. Ainsi, l'une des mesures effectuées dans le cadre de ce travail recourt au questionnaire de Farber. Ce dernier explore les six domaines du modèle SPIKES et satisfait ainsi une validité apparente. Cependant, les autres qualités psychométriques du questionnaire (validité de contenu et surtout de sa validité de construit) ne sont pas vraiment documentées ; les différentes dimensions de sa fidélité, et notamment de sa consistance interne (appréciable, par exemple, grâce au coefficient de Cronbach), ne sont pas non plus avérées. Il est possible en outre que sa traduction en français ait altéré les caractéristiques psychométriques. De fait, nous n'avons pas non plus vérifié les qualités psychométriques de la version traduite. Les mêmes remarques peuvent être formulées à l'égard de la « grille d'Alexander».

Au-delà de ses aspects purement métrologiques, le questionnaire de Farber soulève par ailleurs certaines questions et on pourrait considérer que la conception selon laquelle, pour chacune des vignettes abordées respectivement dans chaque question, il n'existe qu'une seule réponse juste est en partie réductrice, notamment au regard de considérations éthiques ou même simplement psychologiques : les réponses sont très prescriptives et laissent peu de place à la complexité et à l'incertitude. À titre d'exemple (question 11), l'idée qu'il faille sans précaution et sans nuance «tout raconter : diagnostic, traitement, pronostic », sans se donner la possibilité d'étaler les informations dans le temps, fait fi de ce l'on sait des mécanismes de défense qui se mettent en place après l'annonce d'une mauvaise nouvelle (déni, ambivalence, etc.) et qui impliquent éventuellement que l'on étale l'annonce sur la durée, en acceptant des « retours en arrière » de la part des patients.

Enfin, la restriction de l'échantillon étudié aux seuls résidents en stage hospitalier au centre hospitalier universitaire de l'Hôtel Dieu de France de Beyrouth ne permet pas de généraliser sans réserve nos résultats à l'ensemble des résidents inscrits dans 
le tronc commun de médecine interne de l'université Saint-Joseph.

\section{Conclusion et perspectives}

Dans le cadre de cette étude, nous avons pris le parti de tenter d'apporter une démonstration quasi-expérimentale des effets d'un dispositif de formation dédié à l'annonce d'une mauvaise nouvelle. En cohérence avec cette intention, nous avons mis en œuvre une approche psychométrique qui peut ne pas refléter toute la complexité des cadres cliniques réels d'annonce de mauvaises nouvelles. Les effets du dispositif évalué se situent à un niveau taxonomique plus proche de celui connaissances, d'habiletés et de compréhension que d'authentiques compétences. Ces limites étant prises en considération, ce travail peut être considérée comme une étude-pilote, qui montre un effet positif d'une formation à la communication sur les connaissances concernant l'annonce d'une mauvaise nouvelle dans une population de résidents de médecine issus d'une culture arabe. Des démarches pédagogiques plus complexes, telles que celles recourant à l'enseignement en contexte clinique autour de patients réels, en exploitant notamment la puissance des modèles de rôle ou encore les travaux sur la décision partagée, pourraient aussi être envisagées ${ }^{[29,30]}$.

\section{Contributions}

Fady Haddad, Tony Ibrahim et Elie Nemr ont élaboré le protocole de recherche. Tony Ibrahim, Charbel Yazbeck et Elie Haddad ont effectué l'analyse statistique et analysé les données. Georges Maalouly et Tony Ibrahim ont rédigé les versions successives du manuscrit.

\section{Approbation éthique}

Le protocole de l'étude a été approuvé par le comité d'éthique de la Faculté de médecine de
l'Université Saint-Joseph de Beyrouth (Liban) (délibération du 4 mars 2008).

\section{Déclaration d'intérêts}

Les auteurs déclarent qu'ils n'ont pas de conflit d'intérêt financier en lien avec l'objet de cet article. Ils déclarent cependant tous une position hiérarchique à l'égard des étudiants dans le cadre de leurs responsabilités institutionnelles au sein de la faculté, relativement à l'organisation des stages et des enseignements.

\section{Références}

1. Garg A, Buckman R, Kason Y. Teaching medical students how to break bad news. Can Med Assoc J 1997;156:1159-64.

2. VandeKieft GK. Breaking bad news. Am Fam Physician. 2001;64:1975-8.

3. Baile WF, Buckman R, Lenzi R, Glober G, Beale EA, Kudelka AP. SPIKES-A six-step protocol for delivering bad news: application to the patient with cancer. Oncologist. 2000;5:302-11.

4. Orlander JD, Fincke BG, Hermanns D, Johnson GA. Medical residents' first clearly remembered experiences of giving bad news. J GenIntern Med. 2002; 17:825-31.

5. Association of American Medical Colleges. Contemporary Issues in Medicine: Communication in Medicine (Report III of the Medical School Objectives Project). Washington ( DC): AAMC, 1999.

6. International Federation of Medical Students' Associations (IFMSA) and European Medical Students' Associations (EMSA). European Core Curriculum the Students' Perspective. Bristol (UK), 2006.

7. Rosenbaum ME, Kreiter C. Teaching delivery of bad news using experiential sessions with standardized patients. Teach Learn Med 2002;14:144-9.

8. Rabow MW, McPhee SJ. Beyond breaking bad news: how to help patients who suffer. West J Med 1999; 171:260-3.

9. Rosenbaum ME, Ferguson KJ, Lobas JG. Teaching medical students and residents skills for delivering 
bad news: a review of strategies. Acad Med 2004;79:107-17.

10. Park I, Gupta A, Mandani K, Haubner L, Peckler B. J. Breaking bad news education for emergency medicine residents: A novel training module using simulation with the SPIKES protocol. Emerg Trauma Shock 2010;3:385-8.

11. Teike Lüthi F, Cantin B. Annonce de mauvaises nouvelles : une pointe d'EPICES dans l'apprentissage. Rev Med Suisse 2011;7:85-7.

12. Kaplan M. SPIKES: a framework for breaking bad news to patients with cancer.Clin J Oncol Nurs 2010;14:514-6.

13. Alexander SC, Keitz SA, Sloane R, Tulsky JA. A controlled trial of a short course to improve residents' communication with patients at the end of life. Acad Med 2006;81:1008-12.

14. Fallowfield L, Jenkins V, Farewell V, Saul J, Duffy A, Eves R. Efficacy of a Cancer Res UK communication skills training model for oncologists: a randomized controlled trial. Lancet 2002;359:650-6.

15. Razavi D, Delvaux N, Marchal S, Durieux JF, Farvacques C, Hogenraad $\mathrm{R}$ et al. Does training increase the use of more emotionally laden words by nurses when talking with cancer patients? A randomised study. Br J Cancer 2002;87:1-7.

16. Razavi D, Merckaert I, Marchal S, Libert Y, Conradt S, Delvaux N. How to optimize physicians' communication skills in cancer care: results of a randomized study assessing the usefulness of post training consolidation workshops. J Clin Oncol 2003;21:3141-9.

17. Rotter JB. Generalized expectancies for internal versus external control of reinforcement. Psychological Monographs 1966);80(1). (Whole No. 609).

18. Farber NJ, Urban SY, Collier VU, Weiner J, Polite RG, Boyer EG et al. The good news about giving bad news to patients. J Gen Intern Med 2002;17:914-22.

19. Cerclé A., Somat A. Psychologie Sociale. Paris : Dunod, 2005.

20. Back AL, Arnold RM, Baile WF, Fryer-Edwards KA, Alexander SC, Barley GE, Tulsky JA et al. Efficacy of communication skills training for giving bad news and discussing transitions to palliative care. Arch Intern Med. 2007;167:453-60.
21. Quirt CF, Mackillop WJ, Ginsburg AD, Sheldon L, Brundage M, Ginsburg L et al. Do doctors know when their patients don't? A survey of doctor-patient communication in lung cancer. Lung Cancer 1997;18:1-20.

22. Saraiya B, Arnold R, Tulsky JA. Communication skills for discussing treatment options when chemotherapy has failed. Cancer J 2010;16:521-3.

23. Tulsky JA, Fischer GS, Rose MR, Arnold RM. Opening the black box: how do physicians communicate about advance directives? Ann Intern Med 1998;129:441-9.

24. Rothman AI, Cohen R, Bilan S. A comparison of short- and long-case stations in a multiple-station test of clinical skills. Acad Med 1996;71:S110-2.

25. Vaidya VU, Greenberg LW, Patel KM, Strauss LH, Pollack MM. Teaching physicians how to break bad news: a 1-day workshop using standardized parents. Arch Pediatr Adolesc Med 1999;153:419-22.

26. Vu NV, Barrows HS, Marcy ML, Verhulst SJ, Colliver JA, Travis T. Six years of comprehensive, clinical, performance-based assessment using standardized patients at the Southern Illinois University School of Medicine. Acad Med 1992;67:42-50.

27. Andrade AD, Bagri A, Zaw K, Roos BA, Ruiz JG. Avatar-mediated training in the delivery of bad news in a virtual world J Palliat Med 2010;13:1415-9.

28. Razavi D, Delvaux N, Marchal S, Bredart A, Farvacques C, Paesmans M. The effects of a 24-hour psychological training program on attitudes, communication skills and occupational stress in oncology: a randomised study. Eur J Cancer 1993;29A:1858-63.

29. Chamberland M, Hivon R. Les compétences de l'enseignant clinicien et le modèle de rôle en formation clinique. Pédagogie médicale 2005;6:98-111.

30. Franks A. Breaking bad news and the challenge of communication. Eur J Palliat Care 1997;4:61-5.

Correspondance et offprints: Fady Haddad, Département de médecine interne, Hôpital Hôtel-Dieu de France, Ashrafieh, Beyrouth (Liban) et Département de pédagogie médicale, Université Saint-Joseph, Beyrouth, Liban. Télécopie: 9611397772. Mailto : prfghaddad@yahoo.com 


\section{Annexe 1.}

Ce questionnaire comprend 21 questions à choix de réponse. (Réponse attendue en gras)

1- Le temps que vous allouez à l'entretien de mauvaises nouvelles est de :
$\square$ Entre 5-10 min
$\square$ Entre $10-30 \mathrm{~min}$
$\square>30 \mathrm{~min}$
Selon le cas

2- Lors d'une annonce d'une mauvaise nouvelle à un patient, vous recevez un appel téléphonique des urgences. Quelle est votre attitude ?

$\square$ A) Vous répondez au téléphone en interrompant l'entretien ; pour vous c'est un entretien tout à fait comme les autres

$\square$ B) Vous raccrochez et éteignez votre portable

$\square$ C) Vous vous excusez auprès du patient et vous répondez

$\square$ D) Vous lui dites que c'est une urgence et vous quittez le lieu de l'entretien

3- A votre avis, l'endroit idéal où le médecin pourrait annoncer une mauvaise nouvelle serait ?

$\square$ A) Au bureau du médecin ou au lit du malade

$\square$ B) Dans le couloir

$\square$ C) Par téléphone

$\square$ D) Quand l'occasion se présente, quel que soit le lieu

4- Vous êtes en train d'annoncer le diagnostic d'infertilité à un couple, votre attitude serait de :

$\square$ A) Se placer près d'eux sans les toucher

$\square$ B) D'utiliser le contact physique (exemple : le toucher par les mains)

$\square$ C) Préserver la relation médecin malade en se mettant derrière votre bureau

5-Après l'annonce d'une mauvaise nouvelle lors d'un entretien dans votre cabinet, c'est le temps de conclure l'entretien :

$\square$ A) Vous vous levez, vous serrez la main du patient et vous lui demandez d'appeler votre assistant si jamais il a des questions

$\square$ B) Vous faites répéter au patient ce qui s'est déroulé lors de l'entretien pour savoir s'il a bien compris ce que vous avez dit

$\square$ C) Vous lui proposez les options thérapeutiques et fixez d'emblée la date de début du traitement

$\square$ D) Vous leur exposez les modalités du traitement et vous leur laissez le temps pour réfléchir à domicile

6- Vous êtes résident aux soins intensifs et un patient décède d'un arrêt cardiaque. La façon d'annoncer cette nouvelle aux parents :

$\square$ A) Vous leur dites tout carrément : « votre père est décédé ».

$\square$ B) Vous leur dites que le patient a fait un arrêt mais que vous êtes en train de le réanimer depuis 30 minutes et que son cœur n'a pas encore récupéré

$\square$ C) Vous attendez que le médecin de garde vienne annoncer ceci à la famille

$\square$ D) Vous annoncez le décès à la famille tout en leur expliquant les causes du décès et en les rassurant que tous les moyens adéquats de réanimation ont été utilisés

7- Vous êtes en train d'annoncer à la famille d'un malade qu'il a eu un accident vasculaire cérébral hémorragique et vous leur expliquez votre plan thérapeutique quand ils commencent à vous suggérer des modalités thérapeutiques qu'ils ont du entendre d'un autre médecin. Votre attitude serait :

$\square$ A) Vous les interromper et vous imposez votre plan de traitement sans discuter

$\square$ B) Vous les laissez exprimer leur peur et leur angoisse sans que cela n'affecte vos plans

$\square$ C) Vous discutez le plan de traitement et vous exposez des statistiques de survie pour les convaincre

$\square$ D) Vous discutez avec eux dans la perspective de changer le traitement d'une manière qui leur convient 
8- Vous avez posé le diagnostic de leucémie aigüe lymphoblastique chez un garçon de 12 ans. Votre attitude vis-à-vis de l'annonce du diagnostic serait :

$\square$ A) Dire aux parents seulement sans inclure le patient lui-même et laisser le choix aux parents qu'ils lui disent ou non

$\square$ B) Annoncer le diagnostic aux parents et à l'enfant dans le même entretien

$\square$ C) Expliquer la situation aux parents en insistant qu'ils doivent transmettre le message à l'enfant. À défaut, vous le priez

9- Un homme de 40 ans, marié et père de 4 enfants, se présente dans votre cabinet. Après examen clinique et para-clinique, il s'avère qu'il est séropositif HIV. Votre attitude serait :

$\square$ A) Lui annoncer le diagnostic en la présence de sa femme

$\square$ B) Lui annoncer le diagnostic à lui seul et lui laisser le choix de dire ou non à sa famille

$\square$ C) Lui annoncer le diagnostic et l'obliger à le transmette à ses proches

10- Une femme de 70 ans vous consulte pour altération de l'état général. Il s'avère qu'elle a un cancer de l'ovaire à mauvais pronostic. Elle vous questionne sur ce point. Quelle serait votre attitude dans l'entretien : $\square$ A) Lui annoncer le pronostic tout carrément

$\square$ B) Demander à ce que l'un de ses proches soit présent lors de l'entretien

$\square$ C) Lui annoncer le diagnostic sans le pronostic, et si elle insiste vous dites que vous ne savez pas

11- a- Lors de l'annonce d'une mauvaise nouvelle à un homme de 62 ans souffrant d'un myélome multiple, quelle attitude adopterez-vous :

$\square$ A) Lui raconter tout : diagnostic, traitement, pronostic

$\square$ B) Etre plus réservé, utiliser des nuances

$\square$ C) Exiger la présence d'un proche

$\square$ D) Raconter seulement à sa femme

11- b- Et si c'était une femme de 62 ans souffrant d'un myélome multiple, quelle attitude adopteriez-vous ?

$\square$ A) Lui raconter tout : diagnostic, traitement et pronostic

$\square$ B) Etre plus réservé, utiliser des nuances

$\square$ C) Exiger la présence d'un proche

$\square$ D) Raconter seulement à son mari

12- Lors de l'annonce du diagnostic, le patient évoque Dieu dans la conversation :

$\square$ A) Vous prenez en compte vos croyances pour répondre au malade par un langage religieux

$\square$ B) Vous restez objectif sans rien ajouter, vous vous limitez à vos connaissances médicales

$\square$ C) Vous changez de sujet

$\square$ D) Vous prenez ses croyances en compte tout en restant objectif dans votre attitude thérapeutique

13- Un patient passe vous consulter dans votre cabinet. Vous trouvez qu'il souffre d'un cancer du colon métastatique dont le pronostic de survie ne dépasse pas 5 ans. Vous savez que ce malade attend une promotion dans son travail pour devenir directeur national d'une grande chaine et que cette position était le rêve qu'il a tellement voulu accomplir. Que ferez-vous lors de l'annonce du diagnostic :

$\square$ A) Vous lui annoncez le diagnostic avant qu'il ne reçoive la promotion pour débuter le traitement le plus rapidement possible ; d'une part il pourra perdre la promotion mais d'autre part la chance de survie pourrait être améliorée

$\square$ B) Vous lui annoncez le diagnostic après qu'il ait reçu la promotion mais cela va reporter la date du début de traitement

$\square$ C) Vous annoncez la mauvaise nouvelle à un proche dans le but de surseoir à l'annonce jusqu'à la promotion

$\square$ D) Vous lui mentez en lui donnant un diagnostic de « colite inflammatoire » pour débuter le traitement le plus tôt possible avec l'aide d'un proche 
14- Un ami à vous, médecin vous consulte pour altération de l'état général et perte de poids. Aux examens faits vous découvrez qu'il a un carcinome pulmonaire non à petites cellules nécessitant une lobectomie. Quelle serait votre approche envers ce patient :

$\square$ A) Relation amicale affective

$\square$ B) Approche professionnelle médecin/malade

$\square$ C) Approche professionnelle médecin/médecin

15- Une fille de 25 ans célibataire se présente pour douleurs abdominales avec fièvre à 39.5. Le diagnostic de salpingite sévère bilatérale avec formation d'abcès a été posé ; elle pourrait affecter la fertilité de la fille ; vous devez intervenir en urgence par un drainage chirurgical. Votre attitude serait :

$\square$ A) Vous préférez discuter le cas avec les parents en premier pour qu'ils ne vous considèrent pas responsable de l'infertilité mais sans rien dire à la fille pour ne pas la choquer

$\square$ B) Vous annoncez le diagnostic tout de suite à la fille et c'est elle qui va raconter tout à ses parents après l'opération

$\square$ C) Vous annoncez aux parents et à la fille en même temps le diagnostic et le pronostic avant l'opération

$\square$ D) Vous annoncez le diagnostic de salpingite à la fille sans parler du risque d'infertilité et vous la transférez tout de suite en salle d'opération

16- Un homme de 60 ans souffre d'une perte de poids de $5 \mathrm{~kg}$ avec des douleurs abdominales. Il vous consulte et vous posez le diagnostic de cancer du colon. Ce malade, ne voulant pas vous croire, décide de consulter un autre médecin qui lui a formulé le même diagnostic d'une manière différente en parlant d'une « inflammation » à la place de cancer. Ce patient revient chez vous confus. Quelle serait votre attitude :

$\square$ A) Retenir votre diagnostic de début mais cette fois vous prenez votre temps pour le lui formuler d'une façon plus claire en donnant un espoir réaliste.

$\square$ B) Ne rien dire au malade mais contacter l'autre médecin et lui demander strictement de dire la vérité au patient

$\square$ C) Lui demander d'aller se traiter chez l'autre médecin

17- Une patiente atteinte de cancer du sein sous chimiothérapie développe une infection respiratoire ; vous êtes demandés en consultation par son médecin traitant pour traiter cette infection. Elle commence à vous poser des questions sur le pronostic de son cancer quel est votre réponse ?

$\square$ A) Commencer à répondre aux questions en utilisant des statistiques de la littérature même si vous ne savez pas ce que son médecin traitant lui a dit

$\square$ B) Lui dire clairement qu'elle doit poser ces questions à son médecin traitant

$\square$ C) Eviter de répondre à la question en utilisant des termes ambigus : « il y a toujours d'espoir....»

18- Un patient de 50 ans souffrant de cancer du colon métastatique avec mauvais pronostic, est référé à vous par un collègue pour prise en charge. Si vous avez des questions sur les connaissances du patient sur sa maladie, que feriez-vous :

$\square$ A) Vous posez au patient quelques questions pour savoir ce qu'il connait et désire connaitre sur sa maladie

$\square$ B) Vous n'avez pas besoin de savoir ceci et vous commencez à discuter le traitement

$\square$ C) Contacter son médecin durant l'entretien pour vous informez

19- Un homme de 60 ans se présente dans votre cabinet pour fièvre d'origine inconnue. Après avoir demandé les examens para-cliniques, vous trouvez qu'il souffre de lymphome de Hodgkin. Lors de l'annonce de cette mauvaise nouvelle, le patient commence à pleurer. Quelle est votre attitude dans ce cas :

$\square$ A) Vous arrêtez de parler pendant quelques secondes et vous dites après : «je comprend votre réaction, cette nouvelle est dure à apprendre mais il faut y faire face»

$\square$ B) Vous continuez ce que vous étiez en train de dire et exposez le traitement d'une manière scientifique

$\square$ C) Lui dire : «soyez optimiste, les choses pouvaient être pires » 
20- Un patient vous a été référé pour prise en charge d'une fièvre d'origine inconnue ; après des semaines et des séries d'examens le diagnostic de lymphome vasculaire (entité rare) a été posé. Lors de l'entretien et après l'annonce du diagnostic, le patient présente une crise de colère en vous traitant d'incompétent à cause du retard pour poser le diagnostic « de cette catastrophe... » (Selon ses dires). Quelle serait votre attitude :

$\square$ A) Etre strict dans votre conversation; lui dire : « je comprends votre colère, mais retenez vous monsieur ; votre maladie est rare et on n'a pas de tests rapides pour poser ce diagnostic plus tôt »

$\square$ B) Lui demandez de sortir parce que vous ne tolérez pas une discussion avec une personne en colère et les patients dans la salle d'attente vont être angoissés

$\square$ C) Rester indifférent à sa réaction et poursuivre les explications d'une manière professionnelle

21- Un enfant de 14 ans se présente dans votre cabinet pour pollakiurie. A l'examen de sang, le diagnostic de diabète type I se pose. Comment allez-vous lui annoncer cette nouvelle ?

$\square$ A) Tout carrément, vous lui dites : «vous présentez un diabète nécessitant un traitement à base d'insuline à vie » $\square$ B) Vous lui donnez des espoirs : «Vous présentez un diabète nécessitant un traitement à vie mais la médecine est en progrès. Il existe une grande chance de pouvoir transplanter un pancréas dans le futur »

$\square$ C) Vous êtes un médecin réaliste et vous lui dites d'une manière très scientifiques : « le diabète type I entraine une destruction auto-immune du pancréas et nécessite 4 injections d'insuline par jour ». 